\title{
INTRODUCCIÓN
}

\section{La Piedra Natural y el Patrimonio construido: Un mismo campo de investigación}

\author{
R. Fort \\ Instituto de Geología Económica (CSIC-UCM)
}

La piedra natural se puede considerar como uno de los materiales de construcción más tradicionales. El comienzo de su empleo se remonta a la antigüedad, habiendo continuado desde entonces hasta la actualidad. Aunque hoy en día existe una gran variedad de materiales artificiales que compiten en cuanto a sus prestaciones en obra nueva, el uso de la piedra sigue estando altamente valorado y es un material cada vez más solicitado en la construcción. Esto es debido a que los edificios construidos con fachadas de piedra natural no sólo son más representativos, sino también más ecológicos y económicos, principalmente por su mayor durabilidad, ya que su ciclo de vida es en general mucho más largo que el de los materiales artificiales. El comercio mundial de piedra natural se ha incrementado de forma importante desde el año 2001, alcanzando en 2005 un aumento del $107 \%$ las pizarras y piedras similares y del $113 \%$ los mármoles y piedras similares. El volumen de toneladas extraídas de granito sólo experimentó un ligero incremento del 15\% (1). La producción mundial de piedra natural supera las 94.000 .000 de toneladas, muy por encima de las 18.475 .000 toneladas de hace 50 años. Los 10 principales países productores de piedra natural durante 2005 fueron China, India, Irán, Italia, Turquía, España, Brasil, Portugal, Egipto y Grecia.

La evolución de la producción de piedra natural en España se muestra en la Figura 1. El último dato anual publicado corresponde al año 2005, con una producción total de 7.987.000 toneladas, lo que supone un valor de $2500 \mathrm{~m} €$. $(1,2)$. La evolución de la producción total se ha incrementado desde 1995 gracias al importante aumento en la extracción de mármol, la cual ha crecido un $166 \%$ en la última década (2). A pesar de este aumento, la producción nacional ha mostrado un comportamiento distinto al del resto del mundo. Mientras que a nivel mundial se aprecia un incremento importante en los últimos cinco años, como ya se ha indicado, la producción en España se ha estancado desde 2000-2001, incluso disminuyendo la explotación de granitos.

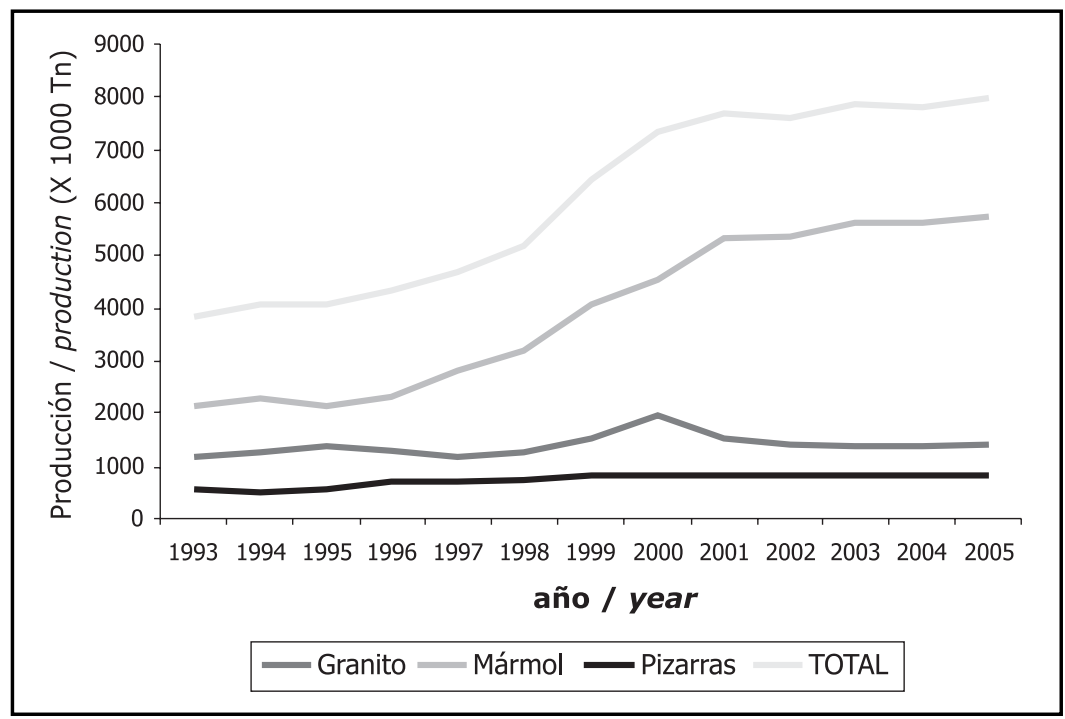

Figura 1. Evolución de la producción de piedra natural en España. Datos tomados de (1) 
La aplicación final de la producción elaborada a nivel nacional se muestra en la Figura 2. En dicha figura se puede apreciar cómo el uso de la piedra en pavimentación alcanza un porcentaje muy alto sobre el resto de los usos.

Parte de esta producción de piedra natural se dedica a la restauración del patrimonio ya construido. No existen datos fiables sobre la utilización de la piedra natural en la conservación de este patrimonio. En España, las inversiones que se realizan en obras de restauración son cada vez más elevadas, cifrándose en 1747 m€ en 2001 (3). Dicha cantidad se incrementa anualmente por encima del IPC. Para este mismo año la producción de piedra natural en España fue de 7.693 .000 toneladas, por un valor de $2.378 \mathrm{~m} €$. (1). Hay que tener presente que la partida que se utiliza para la sustitución de piedra no suele sobrepasar el $1-5 \%$ del valor total de la obra, a no ser que sean proyectos de reconstrucción y/o ampliación de edificios emblemáticos del Patrimonio, o que las intervenciones se localicen en cascos históricos en donde es necesario preservar la estética del conjunto incluyendo no sólo las fachadas sino también la pavimentación de las calles que rodean al bien patrimonial (4).

Aunque existe un gran interés en la utilización de piedra natural en las obras de restauración su uso suele estar limitado por los criterios de restauración establecidos por cartas internacionales de diferente aplicación dentro de cada país.

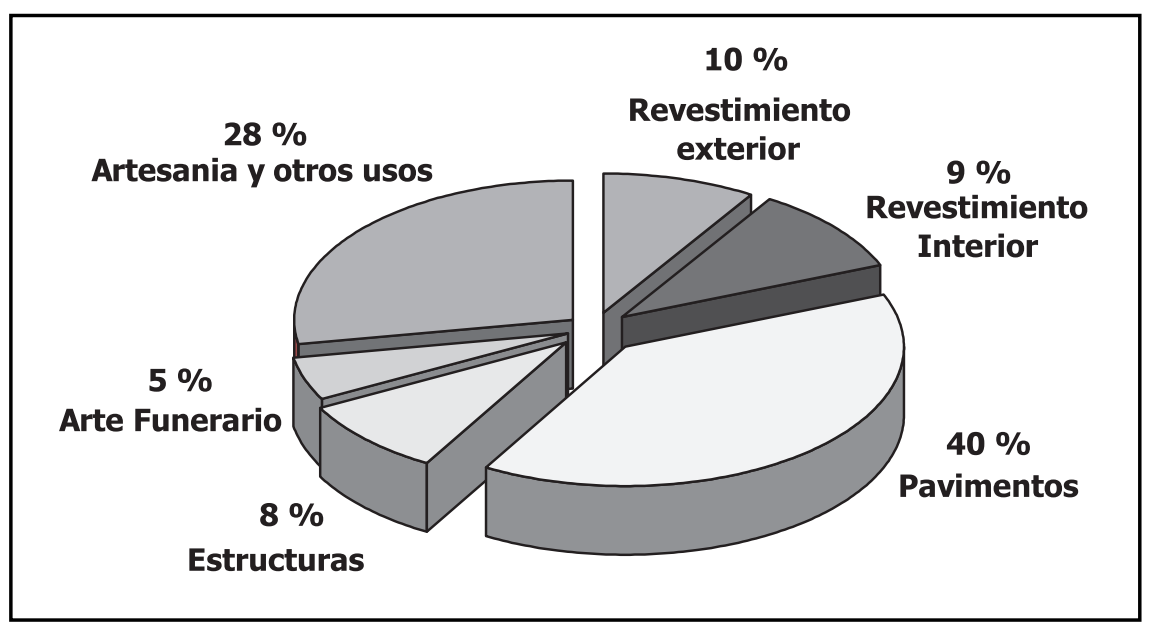

Figura 2. Uso de la piedra naturales España en el año 2005. Datos tomados de (1)

A pesar de la bonanza en la producción mundial de la piedra natural existen mercados nacionales que pueden sufrir más directamente la entrada de nuevos productos que sustituyan a la piedra natural; o que pierdan capacidad competitiva por entrada en el mercado de piedra procedente de otros países. Esto es lo que se observa con la desaceleración de la producción española desde el año 2001, a pesar de ser una época de gran demanda en la construcción. Por este motivo, es necesario mejorar la tecnología de explotación y de elaboración para conseguir un producto de mejor calidad y con un mayor rendimiento. Es por ello importante mejorar las técnicas de corte y acabado, y, sobre todo, es aconsejable investigar sobre la utilización de productos o tratamientos que mejoren la consistencia y durabilidad de la piedra natural frente a los ambientes agresivos a los que va a estar sometida. La piedra natural sufre los procesos agresivos del medio ambiente, sobre todo los de heladicidad y, más aún, los de cristalización de sales. Estos procesos reducen en gran medida la vida media de estos materiales. Igualmente es importante la búsqueda de nuevos recursos naturales que muestren buena calidad desde el origen, así como que respondan a las exigencias para su uso en la construcción.

Igualmente, es necesario ser innovadores en la búsqueda de nuevos usos de la piedra natural. Estos nuevos desarrollos deben encaminarse a la mejora de las cualidades de la piedra, así como a la mejora de las técnicas de corte, elaboración y acabado final de su superficie, sin que ello implique empeoramiento de cualidades y de su durabilidad. La belleza cromática y textural de la piedra natural se incrementará con la disminu- 
ción de su espesor, por lo que su luminosidad por transparencia permitirá su incorporación a diseños arquitectónicos innovadores, junto con sistemas de colocación de piedra natural para fachadas de alta eficiencia y rápida colocación, con una estética acorde a las diferentes tendencias constructivas.

Los acabados superficiales de la piedra natural, tanto de pavimentación como de los aplacados, proporcionan un abanico de posibilidades que otros productos de la competencia no pueden ofrecer. Por ello, dadas las nuevas solicitudes que se realizan sobre este producto en la nueva arquitectura, se debe realizar un gran esfuerzo en la investigación en este campo, de manera que se pueda evitar que la piedra natural quede desmarcada por otros productos artificiales.

Hay que tener presente que la incorporación de nuevos productos a este sector, o una diferente elaboración de la piedra natural, no se debe traducir como un triunfo frente a las técnicas tradicionales. Estas técnicas han demostrado su adecuado funcionamiento en multitud de ocasiones, permitiendo que el patrimonio llegue hasta nuestros días en condiciones muy aceptables. El mercado de la Piedra natural debe abordar con decisión la investigación y el desarrollo en este campo, y sobre todo, aprovechar los conocimientos existentes a nivel científico. Existe un importante campo de investigación que se ha desarrollado a nivel mundial sobre el deterioro de la piedra de construcción utilizada en el patrimonio monumental y arquitectónico, así como sobre las técnicas para su conservación y mejora de su durabilidad ante ambientes agresivos. Los resultados de estas investigaciones pueden ser trasladados al campo de la utilización de la Piedra Natural en construcciones actuales, mejorando con ello los resultados obtenidos. Se sabe cuáles son las características petrofísicas que debe reunir un material para resistir mejor las condiciones adversas de su uso, así como los tratamientos de consolidación o de protección que pueden ser más idóneos, siempre todo ello apoyado con las investigaciones adecuadas.

Las diferentes instituciones encargadas de financiar la Investigación, muestran una inquietud para apoyar toda iniciativa que acerque campos de investigación para las transferencias de sus experiencias y conocimientos a otros campos afines. Un ejemplo de ello es la prioridad que ha marcado la Comunidad de Madrid dentro del IV Plan Regional de Investigación Científica e Innovación Tecnológica (IV PRICIT), en donde una de sus líneas científico-tecnológicas prioritarias es la "Durabilidad de materiales en infraestructuras y edificios. Conservación del Patrimonio". De esta forma, la Comunidad de Madrid apuesta por potenciar los grupos de investigación que desarrollan su trabajo en este campo, en el que la conservación del patrimonio es el objetivo final. El programa Maternas pertenece a esta iniciativa siendo uno de sus objetivos la mejora de la piedra natural ante la experiencia obtenida con el conocimiento del comportamiento de los materiales en el patrimonio arquitectónico.

Igualmente desde el Ministerio de Educación y Ciencia de España se financia, dentro del Programa CONSOLIDER-INGENIO 2010, el programa titulado "Tecnologías para la conservación y revalorización del Patrimonio Cultural". Se intenta consolidar la visión multidisciplinariedad en campo de la conservación de este Patrimonio para poder abordar un problema y alcanzar soluciones más adecuadas en base al avance del conocimiento puesto en común. En este sentido 16 grupos de investigación realizan un trabajo colaborativo en el ámbito del Patrimonio construido cuyos resultados puedan ser transferidos a diferentes sectores de la sociedad y del tejido productivo. En este sentido desde la aproximación de los resultados de la investigación en el patrimonio arquitectónico construido en piedra podrá ser transferido al campo de la investigación y producción de la Piedra Natural.

Esta inquietud también existe en el ámbito de otros países y principalmente dentro de la Unión Europea en donde el VII Programa Marco potencia la interrelación de las diferentes disciplinas. Así, algunas de las investigaciones que están financiando, está relacionadas con la evaluación del deterioro, diagnosis y monitorización para la conservación preventiva y el mantenimiento del patrimonio cultural (ENV.2007.3.2.1.1.), teniendo la piedra natural el importante papel de estar presente en la gran parte de estos bienes patrimoniales sujetos a estudio. 
La plataforma tecnológica de Construcción europea (ECTP) tiene como una de sus áreas de trabajo focalizadas, la relativa al Patrimonio Cultural (Focus Area 6: Cultural Heritage, FACH). En dicho área se agrupa a los profesionales de la industria de la construcción, con los científicos e investigadores de institutos y universidades. La razón de su origen es que cerca del $40 \%$ de las actividades de construcción están centradas en la rehabilitación, reparación y mantenimiento del patrimonio arquitectónico y monumental, en donde, como ya se ha mencionado, la piedra ocupa un lugar destacado como material de construcción.

La revista de materiales de Construcción, sensible a la difusión de las investigaciones en el campo de la construcción, lleva años con esa visión de proximidad entre la piedra de construcción en obra nueva y en el Patrimonio Arquitectónico. En el presente volumen se recogen 18 trabajos, de diferentes especialistas, que abordan desde distintas perspectivas el estudio de la piedra natural ante diferentes situaciones de deterioro y de técnicas de protección.

\section{BIBLIOGRAFÍA}

(1) Roc Máquina: La piedra Natural de España 2007. 544 p. Ed. Reed Business information (2007).

(2) OMPN - Observatorio de Mercado de la Piedra Natural: "Informe sectorial de la piedra natural 2006". Ámbito Nacional. AIDICO (Instituto tecnológico de la construcción) 47 p. En http://www.fdp.es/infsector/docs/informes/informe-sector-pidra-natural-2006.pdf

(3) Alonso, J., Martín Fernández, J.: Preservación del patrimonio histórico de España. Análisis desde una perspectiva económica. 155 p. Fundación Caja Madrid (2004).

(4) Fort, R.: Utilización de la Piedra Natural en Restauración. En: Utilización de rocas y minerales industriales (ed. M. A. García del Cura y J. C. Cañaveras). Seminario de la Sociedad Española de Mineralogía, Vol. 2. 155-182. Ed. Universidad de Alicante (2006). 